\section{AB0696 DETECTION OF COEXISTING MYOSITIS-SPECIFIC AUTOANTIBODIES WITH LINE AND DOT IMMUNOASSAYS IN PATIENTS WITH IDIOPATHIC INFLAMMATORY MYOPATHIES}

Jean-Baptiste Vulsteke ${ }^{1,2}$, Xavier Bossuyt ${ }^{3,4}$, Kristl G. Claeys ${ }^{5,6}$, Doreen Dillaerts ${ }^{4}$, Nele Vanhorebeek ${ }^{7}$, Koen Poesen ${ }^{3,8}$, Jan Lenaerts ${ }^{1}$, Rene Westhovens ${ }^{1,2}$, Philip Van Damme ${ }^{5,9}$, Daniel Blockmans ${ }^{10}$, Petra De Haes ${ }^{11}$, Ellen De Langhe ${ }^{1,2}$. ${ }^{1}$ University Hospitals Leuven, Rheumatology, Leuven, Belgium; ${ }^{2} \mathrm{KU}$ Leuven, Skeletal Biology and Engineering Research Center, Leuven, Belgium; ${ }^{3}$ University Hospitals Leuven, Laboratory Medicine, Leuven, Belgium; ${ }^{4} \mathrm{KU}$ Leuven, Clinical and Diagnostic Immunology, Leuven, Belgium; ${ }^{5}$ University Hospitals Leuven, Neurology, Leuven, Belgium; ${ }^{6} K U$ Leuven, Laboratory for Muscle Diseases and Neuropathies, Leuven, Belgium; ${ }^{7} \mathrm{KU}$ Leuven, Leuven, Belgium; ${ }^{8} \mathrm{KU}$ Leuven, Laboratory for Molecular Neurobiomarker Research, Leuven, Belgium; ${ }^{9} \mathrm{KU}$ Leuven, Department of Neurosciences, Experimental Neurology, VIB Center for Brain and Disease Research, Laboratory of Neurobiology, Leuven, Belgium; ${ }^{10}$ University Hospitals Leuven, General Internal Medicine, Leuven, Belgium;

${ }^{11}$ University Hospitals Leuven, Dermatology, Leuven, Belgium

Background: Myositis-specific autoantibodies (MSAs) can be identified in up to $60 \%$ of patients with idiopathic inflammatory myopathies (IIM) (1). Based on previous immunoprecipitation studies, MSAs are considered to be mutually exclusive (1), but detection of coexisting MSAs with line or dot immunoassay (LIA/DIA) has been described (2-3).

Objectives: To determine the prevalence of detection of coexisting MSAs with different LIAs/DIAs in patients with IIM, assess the concordance between different assays and describe the clinical phenotype of patients with coexisting MSAs.

Methods: Cross-sectional assessment of prevalence of coexisting MSAs as detected by two LIAs (Euroline Autoimmune Inflammatory Myopathies, Euroimmun, Lübeck, Germany; and ImmcoStripe Myositis Advanced LIA, Trinity Biotech, Buffalo, USA) and 1 DIA (12 lgG Dot, Alphadia, Mons, Belgium), concordance between these assays and clinical phenotype of patients with coexisting MSAs in a single-center cohort of patients with a diagnosis of one of the subtypes of IIM as diagnosed by the treating physician.

Results: Nineteen of 145 patients (12\%) had coexisting MSAs on at least one assay: 5 on the DIA (3,5\%) and $5(3,5 \%)$ and $11(7,6 \%)$ on the LIA of Euroimmun and Trinity Biotech respectively. The results were concordant between 2 assays for more than one MSA in only 3 patients. The three combinations of these patients were anti-Jo-1 and anti-NXP-2, anti-Jo-1 and anti-TIF1-gamma, and anti-SAE and anti-NXP2 autoantibodies. The first two patients had an antisynthetase syndrome and the last patient an overlap myositis phenotype. All assays combined, anti-TIF1gamma (9/19), anti-Jo-1 (7/19) and anti-NXP2 (6/19) autoantibodies were the most detected MSAs with concurrent detection of another MSA, though concordance between assays for these MSAs was low to moderate.

Conclusion: Detection of coexisting MSAs with LIA or DIA occurs in a minority of patients with IIM with varying prevalence between assays from different manufacturers. The combination of more than $1 \mathrm{MSA}$ is not concordant between LIAs/DIAs in the vast majority of patients, suggesting that in most patients detection of coexisting MSAs with LIAs/DIAs reflects a problem of specificity of the assays for the involved autoantibodies.

\section{REFERENCES}

[1] McHugh NJ, Tansley SL. Autoantibodies in myositis. Nature Reviews Rheumatology. 2018.

[2] Brouwer R, Hengstman GJD, Egberts V, Ehrfeld H, Bozic B, Ghirardello A, et al. Autoantibody profiles in the sera of European patients with myositis. Ann Rheum Dis. 2001;60:116-23.

[3] Cavazzana I, Fredi M, Ceribelli A, Mordenti C, Ferrari F, Carabellese N, et al. Testing for myositis specific autoantibodies: Comparison between line blot and immunoprecipitation assays in 57 myositis sera. J Immunol Methods. 2016;

Acknowledgement: The study was funded by Alphadia, D-tek, Trinity Biotech and Euroimmun.

Disclosure of Interests: Jean-Baptiste Vulsteke Grant/research support from: SB PhD Fellow at FWO, grant from the Fund Joël Hurlet, Xavier Bossuyt Consultant for: Inova Diagnostics, Kristl G Claeys: None declared, Doreen Dillaerts: None declared, Nele Vanhorebeek: None declared, Koen Poesen: None declared, Jan Lenaerts: None declared, Rene Westhovens Grant/research support from: Bristol-Myers Squibb, Consultant for: Celltrion, Galapagos-Gilead, Philip Van Damme Grant/research support from: Senior clinical investigatorship at FWO-Vlaanderen, Daniel
Blockmans: None declared, Petra De Haes: None declared, Ellen De Langhe: None declared

DOI: 10.1136/annrheumdis-2019-eular.4171

\section{AB0697 LOW DOSE CYCLOPHOSPHAMIDE AND PIRFENIDONE MIGHT WORK IN SYNERGY TO RELIEVE INTERSTITIAL LUNG DISEASE WITH CONNECTIVE TISSUE DISEASE: A PRELIMINARY OBSERVATIONAL STUDY}

Qingran Yan, Lichong Shen, Xiaoxiang Chen. Renji Hospital, Shanghai Jiaotong University School of Medicine, Department of Rheumatology, Shanghai, China

Background: There are anti-inflammation and anti-fibrosis agents available for connective tissue disease associated interstitial lung disease (CTDILD). A clinical study has been initiated to assess the combination of the two (NCT03221257), but whether they can spare dose for each other is unknown.

Objectives: This preliminary study is aimed to observe outcomes of CTDILD patients receiving cyclophosphamide plus pirfenidone as a rescue therapy, with each agent at about one third of routine dosage.

Methods: We enrolled CTD-ILD patients who did not improve their symp toms (dyspnea or cough) after at least one-month steroids treatment (prednisone $\geq 1 \mathrm{mg} / \mathrm{kg}$ daily). Patients who had adjusted immunosuppressive agents other than steroids or had received anti-fibrotic medications within three months before enrollment were ruled out. We switched the treat ment into pulse cyclophosphamide $(0.4 \mathrm{~g} / \mathrm{m} 2$ monthly) combined with pirfe nidone (300mg twice per day). Besides, we reduced the steroids to prednisone $0.5 \mathrm{mg} / \mathrm{kg}$ daily and then tapered routinely. All the patients were followed up for 12 months.

Results: We enrolled seven patients, of whom two had anti-synthetase syndrome, two had Sjögren syndrome, two had scleroderma and one had mixed connective tissue disease. The media DLCO was $51 \%$ of predic tion (range $47.7 \%$ to $63 \%$ ) and media FVC was $72.3 \%$ of prediction (range $39 \%$ to $81 \%$ ). The media 6MWD was $275 \mathrm{~m}$ (range202 to $324 \mathrm{~m}$ ) At the end of 12-month follow-up, all the patients regained functional independence with a media $52.7 \%$ increase of 6 -minute walk distance (range34.4\% - 86.3\%). Pulmonary function tests showed improved forced vital capacity (median improvement $13.4 \%$, range $0-35.9 \%$ ) and DLCO (median improvement $6.3 \%$, range $1.7 \%-16 \%$ ). The HRCT score had a median decrease of $20.1 \%$ (range $11.7 \%$ to $29.6 \%$ ). For quality of life assessment, the SGRQ total score had a median improvement of $53.3 \%$ (range $19.5 \%$ to $61.7 \%$ ). Of note, no adverse events were observed during the 12-month follow-up.

Conclusion: Our Study provided preliminary but promising clinical evidence for a new strategy in treating CTD-ILD, that cyclophosphamide and pirfenidone might work in synergy and spare dose for each other. A well-designed controlled study is needed to further establish its safety and efficacy.

\section{REFERENCES}

None.

Disclosure of Interests None declared

DOI: 10.1136/annrheumdis-2019-eular.2257

\section{AB0697B VALIDATION OF 2017 CLASSIFICATION CRITERIA FOR ADULT AND JUVENILE IDIOPATHIC INFLAMMATORY MYOPATHIES PROPOSED BY EULAR/ACR IN CHINESE PATIENTS}

Xiaohui Zhang, Xinlei Yang, Lanlan Ji, Zhuoli Zhang. Peking University First Hospital, Rheumatology and Clinical Immunology Department, Beijing, China

Background: Idiopathic inflammatory myopathies(IIMs) are heterogeneous disorers characterized by muscle weakness and muscle inflammation Although the Bohan and Peter criteria proposed in 1975 are most widely used $^{[1,2]}$, there are some limitations. Firstly, they did not clearly specify how to exclude other forms of myopathy disease. Secondly, each criterion is not well defined either. EULAR and ACR jointly proposed the classification criteria for adult and juvenile IIMs and their major subgroups in 2017. The data-driven criteria exhibited high sensitivity and specificity. But most of the patients $(62.6 \%)$ in the data were Caucasians, the performance of the criteria in Asian patients is unknown, which was one of the important limitations ${ }^{[6,7]}$.

Objectives: To evaluate the ability of 2017 EULAR/ACR Classification Criteria for Adult and Juvenile Idiopathic Inflammatory Myopathies (IIM) to 
classify IIM in comparison with 1975 Bohan and Peter criteria in Chinese patients.

Methods: Two hundred and twenty-one in-patients with suspected IIM (including 40 children) were retrospectively included in this study. The performance of 2017 EULAR/ACR criteria was evaluated by sensitivity, specificity, positive predictive value, negative predictive value and classification rate, in comparison to the 1975 criteria, with clinical diagnosis as the gold standard.

Results: The sensitivity, specificity, positive predictive value, negative predictive value of the 2017 EULAR/ACR criteria in IIM classification were $92.7 \%, 87.0 \%, 90.1 \%$ and $90.4 \%$ respectively, in contrast to the 1975 Bohan and Peter criteria of $84.0 \%, 52.2 \%, 61.8 \%$, and $77.9 \%$ (Fig 1). The classification rate of 2017 criteria was also much better than that of 1975 criteria $(90.2 \%$ vs. $67.4 \%)$. The performance of the new criteria in general, as well as the new criteria with muscle biopsy was better. Most IIM patients were correctly further subclassified by the classification tree. The positive rate of myogenic lesion in electromyography (EMG), muscular inflammatory edema in magnetic resonance Imaging (MRI) and specific antibodies of myositis were significantly higher in IIM group than those in control group $(\mathrm{p}<0.001$, all).

Conclusion: The 2017 EULAR/ACR criteria exhibited high sensitivity, specificity, classification rate in the Chinese IIM patients, which was superior to the 1975 criteria. The new criteria showed potentials as clinical classification criteria in the future.

Figure 1. Performance of 2017 EULAR/ACR criteria and 1975 Bohan and Peter criteria. $\square$ Sensitivity Specificity
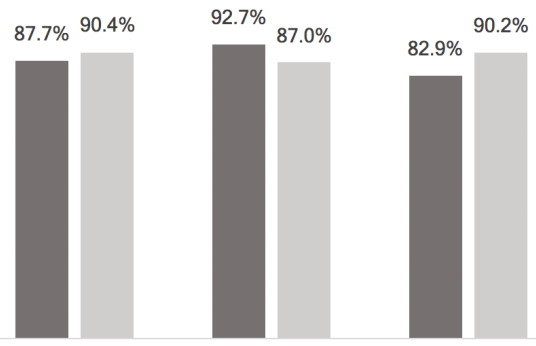

$84.0 \%$

2017 EULAR/ACR 2017 criteria* criteria With muscle biopsy

criteria Without criteria Without
muscle biopsy

* referred to the overall assessment, including patients "score of $\geq 5.5$ without biopsies and score of $\geq 6.7$ with biopsies

\section{REFERENCES}

[1] Bohan A, Peter JB: Polymyositis and dermatomyositis (first of two parts). N Engl J Med 1975, 292:344-347.

[2] Bohan A, Peter JB: Polymyositis and dermatomyositis (second of two parts). N Engl J Med 1975, 292:403-407.

[3] Lundberg IE, Tjarnlund A, Bottai M, Werth VP, Pilkington C, de Visser M, Alfredsson L, Amato AA, Barohn RJ, Liang MH, et al: 2017 European League Against Rheumatism/American College of Rheumatology Classification Criteria for Adult and Juvenile Idiopathic Inflammatory Myopathies and Their Major Subgroups. Arthritis Rheumatol 2017, 69:2271-2282.

[4] Lundberg IE, Tjarnlund A, Bottai M, Werth VP, Pilkington C, Visser M, Alfredsson L, Amato AA, Barohn RJ, Liang MH, et al: 2017 European League Against Rheumatism/American College of Rheumatology classification criteria for adult and juvenile idiopathic inflammatory myopathies and their major subgroups. Ann Rheum Dis 2017, 76:1955-1964.

Disclosure of Interests: None declared

DOI: 10.1136/annrheumdis-2019-eular.2716

\section{AB0697C THE ASSOCIATION OF ANTI-MELANOMA DIFFERENTIATION-ASSOCIATED PROTEIN 5 AND SEASONAL PATTERNS IN ONSET OF IDIOPATHIC INFLAMMATORY MYOPATHIES IN KOREA}

In Seol Yoo, Jinhyun Kim, Seong Wook Kang, Su-Jin Yoo, Seungcheol Shim, Chan Keol Park. Chungnam National University Hospital, Division of Rheumatology, Department of Internal Medicine, Daejeon, Korea, Rep. of (South Korea)

Background: There was some reports of seasonal association with myopsitis onset. With the discovery of new myositis-specific autoantibodies
(MSA), detailed grouping of idiopathic inflammatory myopathies was possible. Therefore, we evaluated the seasonal patterns in the onset of idiopathic inflammatory myositis (IIM) with MSA in Korea.

Objectives: To evaluate the correlation between MSA and seasonal patterns of IIM in Korea.

Methods: A total of 90 patients who met the criteria for probable or definite PM or DM and for whom data were collected from 7 referal centers in korea. 16 myositis-specific autoantibodies were detected by immunoblot with patient's serum. Statistical analyses were performed using a Poisson model that assessed associations of sex, MSA, and month of onset of symptoms or month of diagnosis.

Results: There were no significant seasonal patterns of disease onset in total IIM patients. Among MSAs, anti-synthetase $(n=18)$, anti-transcriptional intermediary factor $1 \gamma($ TIF1 $\gamma)(n=13)$, anti-melanoma differentiation-aasocianted protein 5 (anti-MDA5) $(\mathrm{n}=12)$, and anti-signal recognition particle (SRP) $(n=12)$ were analyzed. Among 55 patients with dermatomyositis, patients with anti-MDA5 showed a significant peak in winter $(n=12$ $P=0.05$ ). This seasonal association was significant in women ( $n=10$ $\mathrm{P}=0.045$ ). Patients with anti-synthetase, anti-TIF1 $\gamma$ or anti-SRP antibodies did not have a significant seasonal onset patterns. There were no significant seasonal patterns in patients without myositis-specific autoantibodies. Conclusion: Patients with anti-MDA5 showed a seasonality of myositis onset, in winter.

Disclosure of Interests: None declared

DOI: 10.1136/annrheumdis-2019-eular.6579

\section{AB0697D CARDIOVASCULAR RISK FACTORS ARE HIGHER IN MALNOURISHED PATIENTS WITH SYSTEMIC SCLEROSIS}

Anna Wojteczek ${ }^{1}$, Marcin Ziętkiewicz ${ }^{1}$, Zbigniew Zdrojewski ${ }^{1}$,

Sylwia Małgorzewicz ${ }^{2} .{ }^{1}$ Medical University of Gdańsk, Department of Internal Medicine, Connective Tissue Diseases and Geriatrics, Gdańsk, Poland; ${ }^{2}$ Medical University of Gdańsk, Department of Clinical Nutrition, Gdańsk, Poland

Background: Cardiovascular disease (CVD) is a well known complication in rheumatoid arthritis and systemic lupus erythematosus. Atherosclerosis (ATS) and its impact on CVD in patients with systemic sclerosis (SSc) still remains unclear. Most of studies are suggesting higher frequency of CVD in SSc in comparison to healthy controls. CVD is a leading cause of death in most developed countries, whereas in SSc population it ranges from 20 to $30 \%$. The etiology of ATS in SSc is unknown. Traditional risk factors, endothelial dysfunction and inflammation can contribute to ATS in SSc population. However, there is lack of data about the impact of the nutritional status on CVD factors in SSc patients.

Objectives: The aim of the study was to determine CVD factors among SSc patients depending on the nutritional status.

Methods: In 55 patients with SSc $(72,7 \%$ well-nourished, $18,1 \%$ malnourished, $9,1 \%$ pre-cachexia) and 49 healthy controls we measured markers of endothelial dysfunction (asymmetric dimethylarginine - ADMA), inflammation (C-reactive protein - CRP, high sensitivity C-reactive protein hsCRP, interleukin 6 - IL-6) and dyslipoproteinemia (oxidized low-density lipoprotein - ox-LDL, high-density lipoprotein- HDL, low-density lipoprotein - LDL and total cholesterol). Nutritional status was determined with subjective global assessment (SGA), body mass index (BMI), bioelectrical impedance analysis (BIA) and anthropometric measurements.

Results: Well-nourished SSc patients had significantly higher level of IL-6 $(6,4 \pm 10,1$ vs. $2,8 \pm 3,6 \mathrm{pg} / \mathrm{ml} ; \mathrm{p}=0,002)$ and lower $\mathrm{HDL}$ cholesterol $(49,9$ $\pm 11,6$ vs. $57 \pm 13,6 \mathrm{mg} / \mathrm{dl} ; \mathrm{p}=0,011$ ) in comparison to healthy control. In malnourished SSc patients there was higher concentration of ADMA $(1,68$ $\pm 0,53$ vs. $1,24 \pm 0,34 \mu \mathrm{mol} / \mathrm{l} ; \mathrm{p}=0.003)$, $\operatorname{CRP}(18 \pm 28,8$ vs. $2,4 \pm 2,5 \mathrm{mg} / \mathrm{l}$; $\mathrm{p}=0.04)$, IL-6 $(21,6 \pm 34,9$ vs. $2,8 \pm 3,6 \mathrm{pg} / \mathrm{ml} ; \mathrm{p}=0.004)$ and lower HDL cho lesterol $(42,5 \pm 18,3$ vs. $57 \pm 13,6 \mathrm{mg} / \mathrm{dl} ; \mathrm{p}=0.01)$ in comparison to healthy control. Pre-cachexia SSc group had significantly lower total cholesterol $(166,8 \pm 28,5$ vs. $219,3 \pm 40,9 \mathrm{mg} / \mathrm{dl} ; \mathrm{p}=0.008)$ and LDL cholesterol $(96,8$ $\pm 21,6$ vs. $139,6 \pm 36 \mathrm{mg} / \mathrm{dl} ; \mathrm{p}=0.007)$. There were no differences in concentration of oxLDL and hsCRP in those groups.

Conclusion: Nutritional status may play role in risk of CVD in SSc patients. Although its contribution to morbidity and mortality rates in CVD in SSc is yet to be established.

Disclosure of Interests: None declared

DOI: 10.1136/annrheumdis-2019-eular.8086 